\title{
Nostalgia como prática? Relendo a pesquisa sobre nostalgia no campo do Marketing
}

\author{
Flávio Medeiros HenRIQUes ${ }^{12}$ \\ Maribel Carvalho Suarez ${ }^{1}$ \\ ${ }^{1}$ Universidade Federal do Rio de Janeiro (UFRJ) / INSTItUto de Pós-GRAduaçÃo e Pesquisa em AdministraçÃo (COPPEAD), \\ RIO DE JANEIRO - RJ, BRASIL

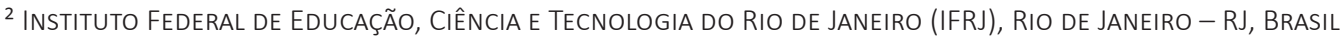

\section{Resumo}

A nostalgia é um poderoso recurso de Marketing e vem apresentando novas formas e dinâmicas no cenário contemporâneo que desafiam suas interpretações clássicas. Considerando que o próprio entendimento do fenômeno do consumo vem sendo revisto, cabe questionar se as explicações atuais sobre nostalgia explicam adequadamente o fenômeno no contexto do consumo. Assim, o objetivo deste trabalho é refletir e propor novas possibilidades de investigação do fenômeno da nostalgia no campo do Marketing a partir das Teorias da Prática. O artigo apresenta duas contribuições principais: ao revisitar a literatura sobre nostalgia no campo, organiza conceitualmente as pesquisas em duas abordagens, a sentimentalista e a cultural. Por fim, o texto também reflete sobre possibilidades de releitura da pesquisa sobre nostalgia a partir das Teorias da Prática.

Palavras-chave: Nostalgia. Teorias da Prática. Consumer Culture Theory.

\section{Nostalgia as a practice? Rereading the research on nostalgia in the field of marketing}

\begin{abstract}
Nostalgia is a powerful marketing resource and has been introducing new forms and dynamics in the contemporary scene that challenge its classic interpretations. Considering that the understanding of the phenomenon of consumption has been revised, it is worth asking whether the current explanations of nostalgia adequately elucidate the phenomenon in the context of consumption. This study proposes new possibilities for investigating the phenomenon of nostalgia in the field of marketing from the perspective of the Practice Theories. The work presents two contributions: by revisiting the literature on nostalgia in the field, it conceptually organizes research into two approaches, sentimental and cultural; by discussing these approaches in light of Practice Theory, we reflect on possibilities for reinterpreting nostalgia research from sociomaterial perspective.
\end{abstract}

Keywords: Nostalgia. Practice Theories. Consumer Culture Theory.

\section{¿Nostalgia como práctica? Releyendo la investi gación sobre la nostalgia en el campo del Marketing}

\section{Resumen}

La nostalgia es un poderoso recurso de Marketing y ha ido introduciendo nuevas formas y dinámicas en la escena contemporánea que desafían sus interpretaciones clásicas. Teniendo en cuenta que se ha cuestionado la comprensión del fenómeno del consumo, cabe preguntarse si las explicaciones actuales sobre nostalgia explican adecuadamente el fenómeno en el contexto del consumo. Así, el objetivo de este trabajo es reflexionar y proponer nuevas posibilidades para investigar el fenómeno de la nostalgia en el campo del Marketing desde las teorías de la práctica. El artículo presenta dos aportes principales: al revisar la literatura sobre la nostalgia en el campo, organiza conceptualmente la investigación en dos enfoques, el sentimental y el cultural. Finalmente, el trabajo también reflexiona sobre las posibilidades de reinterpretar la investigación de la nostalgia desde las teorías de la práctica.

Palabras clave: Nostalgia. Teorías de la práctica. Teoría de la cultura del consumidor. 


\section{INTRODUÇÃO}

Há décadas, a nostalgia é um poderoso recurso de Marketing, capaz de atrair a atenção, o engajamento e o desejo por novos produtos. Nos filmes, nos carros, na música, na moda, nos eventos e destinos turísticos, no design dos eletrodomésticos e nas hashtags \#throwbackthursday que se multiplicam nas redes sociais, entre muitos outros produtos e serviços, o passado faz-se presente como importante dispositivo de mercado. A onda nostálgica que era interpretada no início dos anos 2000 como um efeito passageiro da virada do milênio continua forte e "o interesse dos consumidores pelo passado não demonstra sinais de diminuição" (Brown, 2018, p. 10). Uma reportagem de 2019 do jornal espanhol El País intitulada "O negócio da nostalgia" alertava em sua chamada: "O vinil voltou. Os fliperamas também. O retrô faz sucesso nas bilheterias, e as companhias telefônicas e de videogames relançam seus modelos simbólicos" (Enano, 2019). A própria palavra "nostalgia" parece ter se transformado em uma marca atraente em diferentes contextos de consumo. No YouTube, o Canal Nostalgia, que apresenta vídeos principalmente sobre produtos culturais populares em décadas passadas, como filmes, programas de TV, games etc., conta com 13,1 milhões de inscritos. O álbum mais recente da cantora pop Dua Lipa, denominado Future Nostalgia figura no top 50 da Billboard (Billboard, 2020). Até mesmo uma cor de tinta foi batizada como "verde-nostalgia" por uma importante marca brasileira de tintas (Suvinil, 2020). Exemplos como estes ilustram não somente a força da nostalgia como um fenômeno contemporâneo de Marketing, mas também que ela parece estar tomando novas formas e expressões no cenário do consumo.

O conceito de nostalgia foi originado no século XVII no campo da medicina e deriva das palavras gregas nostos ("volta para casa") e algos ("saudade" ou "dor") para designar uma doença ocasionada pela distância do indivíduo de sua terra natal (Hamilton, Edwards, Hammil, Wagner \& Wilson, 2014). No campo do Marketing, o tema da nostalgia mostra-se presente desde a década de 1990. Questões relacionadas à influência da nostalgia nas preferências do consumidor (Holbrook \& Schindler, 1996), aos tipos de respostas a estímulos publicitários nostálgicos (Stern, 1992) e como a nostalgia cria um senso de identidade do consumidor (Belk, 1990, 1991) figuraram entre os interesses iniciais de pesquisadores de comportamento do consumidor e de cultura e consumo a respeito do fenômeno.

No esforço de renovar suas premissas e sua teorização sobre o fenômeno do consumo, o campo de estudos em cultura e consumo, também conhecido como Consumer Culture Theory (Arnould \& Thompson, 2005), vem presenciando a emergência de novas abordagens que buscam avançar na reflexão sobre as relações entre sujeito-objeto e superar a ênfase dada ao consumidor (agente humano) no processo do consumo. Abordagens diversas como Assemblage Theory (Delanda, 2006), Teoria Ator-Rede (Latour, 1988), Teorias da Prática (Hui, Schatzki \& Shove, 2017), entre outras, vêm sendo adotadas para que se compreenda melhor o papel da materialidade e das relações no processo de consumo (Braga \& Suarez, 2018). Dadas a atualidade e a relevância da nostalgia para o Marketing, conforme argumentado anteriormente, e os novos rumos que o campo vem traçando para compreender o fenômeno do consumo, cabe questionar: as pesquisas atuais sobre nostalgia dão conta de explicar adequadamente o fenômeno em suas diversas manifestações? Considerando que o próprio entendimento do fenômeno do consumo vem sendo questionado pela área, a resposta mais provável é não. Quanto às novas ontologias e abordagens trazidas para o campo do consumo, elas estão sendo utilizadas para buscar novas explicações para o lugar da nostalgia no consumo ou do consumo na nostalgia? Como será apresentado, a literatura sinaliza que a resposta para essa pergunta também é não.

Assim, o objetivo deste trabalho consiste em refletir e propor novas possibilidades de investigação do fenômeno da nostalgia no campo do Marketing a partir das Teorias da Prática (Hui et al., 2017; Shove, Pantzar \& Watson, 2012). Para isso, o trabalho revisita a literatura sobre nostalgia no campo do comportamento do consumidor, de modo a compreender como esta é conceituada e investigada, para então refletir sobre conceitos das Teorias da Prática que podem se somar ou confrontar as premissas das atuais investigações sobre nostalgia.

Além de contribuir com os estudos sobre o tema ao organizar conceitualmente as pesquisas atuais em duas abordagens teóricas, a sentimentalista e a cultural, o trabalho também propõe releituras possíveis da nostalgia por meio de exemplos que ilustram possibilidades de aplicação das premissas e conceitos das Teorias da Prática para o estudo do fenômeno no campo do comportamento do consumidor. Para a elaboração dessas contribuições, o trabalho apresenta, após esta introdução, as origens e a evolução do conceito de nostalgia, na segunda seção. Na terceira seção, são propostas e discutidas duas perspectivas conceituais sobre nostalgia adotadas no campo do Marketing: a sentimentalista e a cultural. A quarta seção analisa criticamente as duas perspectivas de estudo da nostalgia predominantes no campo, discutindo suas limitações e contribuições. A quinta seção apresenta o arcabouço conceitual básico das Teorias da Prática. A sexta seção propõe e discute possibilidades de releitura da nostalgia através da lente das Teorias da Prática. Por fim, apresentam-se as implicações, limitações do estudo e reflexões para auxiliar pesquisadores nos trabalhos futuros sobre nostalgia. 


\section{Sobre as origens e a evolução do conceito de nostalgia}

Os primeiros esboços sobre a natureza da nostalgia deram-se no âmbito da medicina, sendo que o primeiro relato científico conhecido sobre o tema aparece na dissertação médica de Johannes Hofer, publicada em 1688 (Boym, 2001). Em sua dissertação, o médico suíço descreve a nostalgia como uma doença típica dos marinheiros e soldados que viajavam para terras distantes. Entre seus principais sintomas, estariam a náusea, a perda de apetite, as alterações nos pulmões, a inflamação cerebral, as paradas cardíacas, a febre, o marasmo e a propensão ao suicídio (Anspach, 1934). Desse modo, os primeiros estudos sobre a nostalgia definiam como característica central dessa "doença" o deslocamento espacial do indivíduo.

O insucesso da medicina em curar a doença da nostalgia despertou, na era industrial, o olhar das Ciências Sociais, que passou a testemunhar uma epidemia de nostalgia em nível de sociedade, fomentando a construção de museus, a restauração de monumentos e tradições e o nascimento de uma arte romântica que buscava resgatar um passado idealizado como puro e desacelerado. Esse crescente movimento de institucionalização da nostalgia (Garrido \& Davidson, 2019) passou a ser predominantemente interpretado no campo das Ciências Sociais, já na primeira metade do século XX, como um sentimento e um discurso tipicamente conservador emergente da noção de irrecuperabilidade de um passado glorioso destruído pela modernidade. Tal associação levou o tema a ser tratado como algo puramente antiprogressista e alienante, despertando, dessa forma, mais a crítica do que a curiosidade dos pesquisadores (Boym, 2001; Gehler, 2017; Pickering \& Keightley, 2006).

No campo da Psicologia, a conotação negativa construída em torno da nostalgia como uma "doença da mente" predominou até a metade do século XX. Novas interpretações foram fornecidas pelo sociólogo Fred Davis na segunda metade do século XX ao descrever a nostalgia como um sentimento "agridoce", que traz tanto consequências negativas quanto positivas para o indivíduo (Routledge, 2016). No entanto, ainda no cenário contemporâneo, no campo da Psicologia, predomina a interpretação de que a nostalgia é desencadeada por sentimentos de solidão, depressão, descontinuidade e descontentamento com o presente, talvez devido às interpretações da Medicina e das Ciências Sociais do início do século (Garrido \& Davidson, 2019). Conforme observa Routledge (2016), o campo da Psicologia possuía, até a última década, um volume insípido de pesquisas empíricas que buscassem compreender melhor o fenômeno da nostalgia e suas consequências. $\mathrm{O}$ autor observa que a maioria dos estudos empíricos atuais sobre nostalgia provém dos estudos do consumo e do comportamento do consumidor. Isso reforça a importância acadêmica da temática para a área de Marketing.

Os estudos sobre a nostalgia no campo do Marketing iniciaram-se, principalmente, na última década do século XX, explorando sua influência nas preferências do consumidor (Holbrook, 1993), os tipos de respostas à publicidade nostálgica (Stern, 1992) e a relação entre nostalgia e construção de identidade do consumidor (Belk, 1990, 1991). Após a virada do milênio, a nostalgia continuou demonstrando-se promissora como tema de investigação, inspirando estudos sobre seu uso como estratégia de Marketing (Brown, Kozinets \& Sherry, 2003), sobre as fontes da nostalgia na experiência de consumo (Goulding, 2001) e sobre sua capacidade de articular o consumo para criar passados utópicos (Maclaran \& Brown, 2005). Estudos mais recentes exploram temáticas como a construção de mercados nostálgicos (Brunk, Giesler \& Hartmann, 2018) e o papel do mercado em modificar a nostalgia por meio da continuidade (Cross, 2017) e apresentam várias questões que demonstram que a nostalgia é um fenômeno de grande relevância no contexto do consumo e que não se tratava de mero modismo ou onda sentimental desencadeada pela virada do milênio (Brown, 2018).

As Ciências Sociais também parecem ter reconhecido recentemente a relevância do fenômeno. Pickering e Keightley (2006) questionam interpretações clássicas que caracterizam a nostalgia como oposto binário da história e antítese da utopia. Para os autores, a nostalgia não é apenas uma busca por segurança ontológica no passado, mas também um mecanismo social útil de orientação frente às incertezas do presente, quando utilizada criticamente. Esse uso crítico da nostalgia teria como preocupação central o surgimento de um novo modo de se relacionar com o passado na modernidade. No campo da Psicologia Social, Routledge (2016) criou um programa de pesquisa para investigar empiricamente os aspectos positivos e negativos da nostalgia e identificou que esta aumenta estados psicológicos positivos, como humor, sentimentos de conexão social, autoestima, autocontinuidade e percepções de significado na vida, além de atuar como mecanismo regulador de estados psicológicos negativos, como a angústia e a solidão. Tais resultados, conforme observa o autor, confrontam a visão predominante no campo da Psicologia de que a nostalgia é um fenômeno predominantemente negativo e alienante. No campo da Antropologia, o crescimento do interesse pelos estudos de Memória na primeira década do milênio levaram a um crescimento do interesse pela nostalgia, sendo esta conceituada como uma prática cultural heterogênea, com formas, significados e efeitos que mudam de acordo com o contexto, além de ocorrer apenas em temporalidades ontológicas específicas (Angé \& Berliner, 2015). 
Enfim, a nostalgia vem despertando o interesse de diversos campos do saber, sendo que alguns destes somente agora começam a se preocupar em compreender sistematicamente o fenômeno e suas consequências. A área de Marketing, por outro lado, vem desenvolvendo pesquisas sobre a relação entre nostalgia e consumo há cerca de 30 anos. Dado o histórico de investigações sobre o tema no campo, uma reflexão importante diz respeito às bases teóricas utilizadas pelos pesquisadores de comportamento do consumidor para conceituar nostalgia. $O$ tópico a seguir se dedica a essa questão.

\section{Perspectivas conceituais sobre a nostalgia no campo do Marketing}

Antes de propor novas possibilidades de releitura da nostalgia a partir das premissas e conceitos das Teorias da Prática, realizou-se um esforço para identificar como o Marketing conceitua nostalgia, refletindo-se sobre trabalhos recentes e seminais do campo e identificando os autores de outras áreas que influenciam direta ou indiretamente na formação das premissas utilizadas para conceituar o fenômeno. Tal revisão da literatura, que pretendeu apenas rastrear os conceitos, autores e premissas sobre nostalgia em estudos do comportamento do consumidor, permitiu identificar duas perspectivas conceituais sobre a nostalgia existentes no campo: 1) a perspectiva sentimentalista, mais alinhada com os estudos seminais dos anos 1990 sobre a relação entre consumo e nostalgia, que a conceitua como um fenômeno predominantemente cognitivo; e 2) a perspectiva cultural, emergente a partir da virada do milênio e que compreende a nostalgia como um fenômeno cultural, com contornos contextuais. O Quadro 1 apresenta os principais autores e premissas de cada uma das perspectivas, além de exemplificar temáticas estudadas em cada uma delas.

Quadro 1

Quadro sintético das perspectivas sentimentalista e cultural

\begin{tabular}{|c|c|c|c|}
\hline Categoria & $\begin{array}{c}\text { Autores das } \\
\text { Ciências Sociais }\end{array}$ & Premissas & Exemplos de pesquisas no campo do Marketing \\
\hline $\begin{array}{c}\text { Perspectiva } \\
\text { sentimentalista }\end{array}$ & $\begin{array}{c}\text { Fred Davis (1979) } \\
\text { Colin Campbell } \\
\text { (1987) } \\
\text { Grant McCracken } \\
\text { (1988) }\end{array}$ & $\begin{array}{l}\text { 1) A nostalgia trata-se de um sentimento } \\
\text { individual ou coletivo de orientação retros- } \\
\text { pectiva, emergente da confrontação entre } \\
\text { presente e passado. } \\
\text { 2) A nostalgia é uma resposta cognitiva } \\
\text { universal desencadeada por determinados } \\
\text { estímulos independentemente do contexto. } \\
\text { 3) No contexto do consumo, a nostalgia } \\
\text { resulta do deslocamento de significados de } \\
\text { perfeição para um passado idealizado, sendo } \\
\text { que os objetos atuam como pontes materiais } \\
\text { para essas dimensões idealizadas. }\end{array}$ & $\begin{array}{l}\text { - Efeitos dos apelos publicitários nostálgicos } \\
\text { (Khoshghadam, Kordrostami \& Liu-Thompkins, 2019; } \\
\text { Muehling, Sprott \& Sultan, 2014). } \\
\text { - Nostalgia como recurso psicológico e social (Kessous, } \\
\text { Roux \& Chandon, 2015; Lasaleta, Sedikides \& Vohs, 2014). } \\
\text { - Efeitos sobre conexão com marcas (Davari, Iyer \& } \\
\text { Guzmán, 2017; Ford, Merchant, Bartier \& Friedman, 2018). } \\
\text { - Nostalgia e busca de autenticidade (Goulding \& } \\
\text { Derbaix, 2019). } \\
\text { - Nostalgia e preferências do consumidor (Loveland, } \\
\text { Smeesters \& Mandel, 2010; Zhou, Wang, Zhang \& } \\
\text { Mou, 2013). }\end{array}$ \\
\hline $\begin{array}{c}\text { Perspectiva } \\
\text { cultural }\end{array}$ & $\begin{array}{l}\text { Svetlana Boym } \\
\qquad(2001) \\
\text { Fredric Jameson } \\
\text { (1991) }\end{array}$ & $\begin{array}{l}\text { 1) A nostalgia é um fenômeno cultural sensível } \\
\text { às representações sobre o tempo, orientado } \\
\text { simultaneamente para o passado, o presente } \\
\text { e o futuro. } \\
\text { 2) A nostalgia apresenta-se de diferentes } \\
\text { modos de acordo com o contexto, ocorrendo } \\
\text { apenas em determinadas temporalidades } \\
\text { ontológicas. } \\
\text { 3) No contexto do consumo, a nostalgia não } \\
\text { somente exerce efeitos sobre o consumo e } \\
\text { os mercados, como também é modificada } \\
\text { por eles. }\end{array}$ & $\begin{array}{l}\text { - Nostalgia e modificação de categorias culturais } \\
\text { (Cross, 2017). } \\
\text { - Nostalgia como fenômeno epocal pós-moderno } \\
\text { (Armstead \& McKinney, 2019; Higson, 2014). } \\
\text { - Emergência de mercados e nostalgias em contextos } \\
\text { de mudança (Brunk et al., 2018). } \\
\text { - Nostalgia como crítica e luta social (Cervellon \& } \\
\text { Brown, 2018). }\end{array}$ \\
\hline
\end{tabular}

Fonte: Elaborado pelos autores. 


\section{A perspectiva sentimentalista}

A perspectiva sentimentalista apresenta como característica principal a compreensão da nostalgia como um sentimento relacionado à biografia de indivíduos ou grupos, sendo este desencadeado predominantemente por memórias positivas da juventude. Tal perspectiva é a mais antiga e que concentra a maioria das pesquisas no campo, tendo seu início nos anos 1990. Três autores das Ciências Sociais parecem ter importância fundamental para a formação de um conjunto de premissas sobre a nostalgia no campo do Marketing, as quais continuam sendo adotadas em estudos recentes: Fred Davis, Colin Campbell e Grant McCracken.

O livro Yearning for Yesterday: A Sociology of Nostalgia, de Fred Davis, publicado em 1979, aparece como a referência mais comum na conceituação de nostalgia. Os estudos contemporâneos que adotam tal perspectiva, por exemplo, Khoshghadam et al. (2019) e Huang, Huang e Wyer (2016), normalmente citam pesquisas seminais da área como as de Holbrook e Schindler (1991), Stern (1992) e Belk $(1990,1991)$ para conceituar nostalgia. Esses trabalhos seminais do campo, por sua vez, conceituam a nostalgia com base na ideia de que o fenômeno se trata de um sentimento misto de prazer e dor em relação a um passado individual ou coletivo idealizado, apoiados no referido livro de Davis (1979). Em outras palavras, as obras seminais do campo do Marketing da década de 1990, citadas pela maioria dos estudos atuais da perspectiva sentimentalista, partem predominantemente de um mesmo autor para conceituar nostalgia.

O conceito de nostalgia de Davis (1979), por sua vez, alinha-se a dois outros conceitos de grande relevância nos estudos do consumo, o que pode explicar sua ampla adoção. O primeiro conceito é o daydream, de Colin Campbell. Segundo Campbell (1987), o daydream refere-se a uma construção imaginativa de significados de perfeição, os quais são perseguidos materialmente por meio do consumo. Sua finalidade não seria necessariamente a aquisição e o uso de um objeto, mas o prazer de imaginar suas potencialidades na esfera dos significados, pois as imperfeições da realidade empírica quebrariam o encanto que o objeto possui na esfera da imaginação. O segundo conceito é o "deslocamento de significados", de Grant McCracken. De acordo com McCracken (1988), a ideia de que o mundo contemporâneo é percebido como essencialmente inautêntico faz com que o consumidor desloque seus ideais e expectativas para dimensões idealizadas de tempo e espaço, buscando acessá-las materialmente por meio dos objetos. Em comum, tais conceitos ajudam a fundamentar a noção de que a nostalgia se manifesta na confrontação entre real e imaginário, sendo os objetos portadores de significados idealizados, o que parece ter criado terreno fértil para o nascimento de uma concepção cognitivista.

Dos autores de Marketing, o conceito que se destaca em termos de frequência é o de Holbrook e Schindler (1991, p. 330), segundo o qual a nostalgia seria "uma preferência em relação a objetos que eram comuns na juventude de um indivíduo". Belk (1990, p. 670), para quem a nostalgia é "um estado melancólico induzido por um objeto, uma cena, um cheiro ou tipo de música", e Holak e Havlena (1998, p. 218), para os quais a nostalgia é "um complexo sentimento, emoção ou estado de humor com valência positiva produzido pela reflexão do indivíduo sobre coisas associadas com o passado", também são citados, com menor frequência. Vale ressaltar que tanto Holbrook e Schindler (1991) e Belk (1990) quanto Holak e Havlena (1998), apesar de destacarem nuances diferentes da nostalgia em suas conceituações, citam Davis (1979) para fundamentarem suas concepções sobre o fenômeno.

Trabalhos recentes da perspectiva sentimentalista, de modo geral, parecem ter maior preocupação em compreender como a nostalgia afeta o consumidor e as relações de consumo do que propriamente discutir a nostalgia em si. Por exemplo, Lasaleta et al. (2014) concluíram que a nostalgia leva ao comportamento de caridade, uma vez que sensibiliza o consumidor a diminuir seu apego pelo dinheiro diante de uma conexão simbólica com um "outro" relevante que compartilha um passado comum. Kessous et al. (2015) identificaram que os consumidores são mais propensos a colecionar e presentear pessoas amadas com produtos de marcas percebidas como nostálgicas. Areni (2019) concluiu que o envolvimento do consumidor com conteúdo de mídia social que evoca nostalgia atende à necessidade de segurança ontológica. Ford et al. (2018, p. 19) criaram o conceito de nostalgia da marca como o "reflexo do passado composto por memórias, emoções e pensamentos relacionados às experiências vividas ou idealizadas do consumidor com a marca".

Enfim, os pesquisadores da perspectiva sentimentalista, apesar de criarem novas variações conceituais sobre a nostalgia, parecem compartilhar as seguintes premissas em suas investigações: 1) a nostalgia trata-se de um sentimento individual ou coletivo de orientação retrospectiva, emergente da confrontação entre presente e passado; 2) a nostalgia é uma resposta cognitiva universal (ou seja, independe do contexto) desencadeada por estímulos predominantemente mnemônicos; 3) no contexto do consumo, a nostalgia resulta do deslocamento de significados de perfeição para um passado idealizado, 
sendo que os objetos atuam como pontes materiais para essas dimensões idealizadas, combinando o prazer da tentativa de retorno com a frustração do reconhecimento da irrecuperabilidade do passado.

Em geral, os trabalhos dessa perspectiva, apesar de serem mais numerosos e constituírem uma vertente mais antiga no campo, dedicam mais esforço para testar diferentes efeitos da nostalgia sobre o consumidor, assumindo as noções conceituais seminais do campo, do que para esboçar alguma nova tentativa de compreender e conceituar o que ela é. O único esforço de revisão da nostalgia dentro da perspectiva sentimentalista decorre de estudos que investigam os aspectos individual e socialmente positivos da nostalgia (Kessous et al., 2015; Lasaleta et al., 2014; Zhou, Wildschut \& Sedikides, 2011). Desse modo, confronta-se a noção cristalizada no campo da Psicologia de que a nostalgia é um sentimento predominantemente negativo, melancólico e alienante (Routledge, 2016). Contudo, ainda assim concebe-se a nostalgia como um fenômeno cognitivo.

\section{A perspectiva cultural}

A perspectiva cultural é relativamente mais recente no campo de Marketing, apresentando, como consequência, menor número de pesquisas. Sua principal característica é investigar não somente como a nostalgia afeta o consumo, mas como o consumo e o sistema de Marketing também a modificam. Dois autores das Ciências Sociais destacam-se em fornecer as premissas para essa perspectiva: Svetlana Boym e Fredric Jameson.

A historiadora Boym (2001), em seu livro The Future of Nostalgia argumenta que, apesar de a nostalgia ser amplamente estudada como um fenômeno médico, ela é uma emoção histórica nascida e propagada com o romantismo e com a cultura de massa. Seria, assim, transformadora da sociedade e transformada pelas mudanças históricas, culturais e tecnológicas. Para Boym, a nostalgia é um fenômeno antes estrutural do que individual. O referido livro de Boym é a fonte das Ciências Sociais mais citada por autores dessa perspectiva.

Outro autor citado por pesquisadores dessa perspectiva é Fredric Jameson. Dele, cita-se, sobretudo, o livro Postmodernism, or, the Cultural Logic of Late Capitalism, de 1991, sendo o principal elemento adotado de sua obra a ideia de que a nostalgia na contemporaneidade é um sintoma da condição pós-moderna. Tal nostalgia, segundo Jameson (1991), seria atemporal ou sobre "um passado fora do tempo" (Higson, 2014), que convive com o presente e se manifesta mais como uma preferência estética do que como sentimento (Brown, Hirschman \& Maclaran, 2001). Tanto a leitura de Boym quanto de Jameson trabalham com a noção de que a nostalgia é um fenômeno estrutural e dependente do contexto histórico.

Uma das principais preocupações dos pesquisadores dessa perspectiva é compreender como o sistema de Marketing e a tecnologia interagem com a nostalgia na contemporaneidade. Higson (2014), em pesquisa sobre consumo da nostalgia em filmes e sites da internet, descreve esta como um importante estímulo para a nostalgia, visto que a disponibilidade de imagens e representações do passado no ambiente on-line cria um imenso repertório simbólico para o consumidor. $\mathrm{O}$ autor ainda verificou em seu estudo que o consumidor não simplesmente responde a um estímulo nostálgico, mas possui papel ativo na criação da nostalgia ao engajar-se em um texto e modulá-lo de acordo com seus próprios interesses. Cross (2017) analisou a nostalgia contemporânea a partir do estudo de coleções de brinquedos e observou que a velocidade nas mudanças nos bens criou uma forma de nostalgia mais associada a um passado recente do que a um passado distante. $\mathrm{O}$ autor identificou que o sistema de Marketing possui papel fundamental na criação de uma nova relação entre o consumidor, suas coleções e seus sentimentos. Em seu estudo sobre coleções de brinquedos, Cross (2017) verificou que nostalgia fomentou o nascimento de um mercado de coleções de brinquedos, ajudando no processo de legitimação da ideia de que adultos também podem consumir esses objetos antes associados exclusivamente à infância. Tal legitimação, por sua vez, levou novas gerações de consumidores a comprar e utilizar brinquedos continuamente entre a infância e a idade adulta, transformando a descontinuidade que dava origem à nostalgia em uma continuidade. Em outras palavras, a nostalgia fora a fonte da própria destruição, nesse caso.

Tal perspectiva também se alinha com os debates recentes sobre nostalgia nas Ciências Sociais, buscando compreender a nostalgia como um fenômeno estrutural e dependente do contexto, que ocorre apenas em determinadas temporalidades ontológicas (Angé \& Berliner, 2015). Brunk et al. (2018), por exemplo, identificaram formas e dinâmicas específicas da nostalgia dentro do contexto de mudança de regime político e econômico na Alemanha após a queda do muro de Berlim, como sua influência na naturalização da hegemonia capitalista através da ressignificação de memórias sociais. Os autores identificaram a emergência de três tipos de nostalgia pelo socialismo em momentos de insatisfação com o novo sistema capitalista, que, ironicamente, levaram à emergência de novos mercados de produtos nostálgicos contra o capitalismo, destacando que a nostalgia toma formas específicas em diferentes contextos. 
A perspectiva cultural também aborda a nostalgia como um fenômeno orientado simultaneamente para o passado, o presente e o futuro, e não uma confrontação sentimental entre passado e presente. Cervellon e Brown (2018), por exemplo, demonstram como as consumidoras francesas do estilo neoburlesco utilizam recursos do passado de modo irônico para criar contrastes entre um passado de submissão ao machismo e um presente de luta social, de modo a construir condições sociais para um futuro mais livre. Tal premissa condiz com o argumento de Pickering e Keightley (2006) de que a nostalgia, ao contrário de ser um oposto negativo da utopia, é um mecanismo não só de produção, mas também de uso da memória social para construir futuros desejados a partir da crítica do presente.

Com recortes mais recentes, a perspectiva cultural encontra-se em construção. Os textos apresentados nesta seção não compõem necessariamente uma visão coesa sobre nostalgia. Contudo, tais trabalhos possuem em comum interpretações e discussões que desafiam a adequação e a completude da perspectiva sentimentalista da nostalgia. Tais trabalhos priorizam uma perspectiva que não restringe a nostalgia a um sentimento, mas a abordam como um fenômeno cultural contemporâneo resultante: 1) da emergência de novas representações culturais sobre o tempo; 2) das ações do sistema de Marketing; e 3) das possibilidades criadas pelas novas tecnologias. Nesse sentido, esses estudos descortinam novas possibilidades de se enquadrar o fenômeno da nostalgia.

Em resumo, podemos apontar três tipos de premissas da perspectiva cultural que desafiam as noções utilizadas pela perspectiva sentimentalista para conceituar a nostalgia: 1) a nostalgia é um fenômeno cultural sensível às representações sobre o tempo, orientado simultaneamente para o passado, o presente e o futuro; 2 ) a nostalgia apresenta-se de diferentes modos de acordo com o contexto, ocorrendo somente em determinadas temporalidades ontológicas; 3 ) no contexto do consumo, a nostalgia não somente exerce efeitos sobre o consumo e os mercados, como também é modificada por eles. Tendo delineado distinções conceituais em torno das duas abordagens presentes na investigação da nostalgia, o tópico a seguir procura apontar caminhos, limitações e provocações às duas perspectivas identificadas.

\section{Contribuições, limitações e provocações às perspectivas sentimentalista e cultural}

Conforme discutido na seção anterior, a perspectiva cultural ainda parece apresentar importantes possibilidades de releitura da nostalgia no campo do Marketing, uma vez que traz premissas e interpretações que desafiam e ampliam a noção de nostalgia sentimentalista, predominante entre os pesquisadores de Marketing. $O$ enfoque nas mudanças históricas, sociais e tecnológicas, bem como no papel do sistema de Marketing, trouxe ao campo uma compreensão da nostalgia também como um fenômeno cultural. Nesse sentido, ainda cabem estudos que respondam: como o sistema de Marketing modificou-se historicamente no sentido de aceitar como legítima a ideia de lançar produtos vintage? Como a noção de "objeto ultrapassado" se transforma em objeto "retrô"? Como as práticas de estetização de objetos do passado ganharam legitimidade no discurso do Marketing? Questões como estas podem gerar insights sobre a relação entre os sistemas de Marketing e a transformação das representações temporais do consumidor.

Outro ponto interessante descrito nos estudos da seção anterior refere-se às possíveis transformações da relação pessoa-objeto pela tecnologia. Como a tecnologia, ao criar um repertório representacional sobre o passado tão grande e acessível, modifica as relações afetivas entre consumidor e objeto? Como a recuperabilidade do passado no presente e a fusão destas categorias modifica as relações entre o consumidor e o objeto? Seria essa vivência que funde passado e presente sem sentimento um tipo de nostalgia? Ou o conceito de nostalgia deve restringir-se a um sentimento de prazer e dor diante do irrecuperável? Se sim, que construto descreve esse fenômeno de vivência do passado-presente em que a preferência estética predomina sobre o sentimento (Brown et al., 2001)? A delimitação do que é nostalgia diante da identificação de novas articulações temporais presentes na relação pessoa-objeto, nesse sentido, permanece em aberto.

Em relação às categorias que localizam um objeto no tempo, por exemplo, questionando se este é do passado ou contemporâneo, se é "anos 1970" ou "anos 1990", a perspectiva cultural também instiga questionar: como a constante reciclagem de imagens e objetos do passado no presente afeta a categorização temporal dos objetos? Em outras palavras, se um objeto do passado é relançado no mercado e se torna popular em outro tempo, seria esse objeto ainda percebido como "antigo" por gerações mais jovens que não sabem de seu relançamento? Seria possível a enxurrada de relançamentos criar uma imprecisão no reconhecimento daquilo que é efetivamente novo ou relançamento? Seria irônica a coexistência entre pessoas e objetos do passado se estes não são reconhecidos como passado, pois assim seria inviável a evocação da nostalgia sentimental. Ou seja, seria possível que os mercados vintage e a tecnologia destruíssem a consciência temporal de produtos? 
Ainda que as duas perspectivas identificadas sinalizem caminhos férteis (em especial, a perspectiva cultural) para novas compreensões sobre a relação entre nostalgia e consumo, podemos refletir criticamente sobre algumas de suas limitações. Nosso esforço de identificação das bases conceituais da nostalgia no campo sinaliza a fragilidade teórica do conceito de nostalgia adotado pela perspectiva sentimentalista, uma vez que a maioria dos estudos utiliza praticamente as mesmas fontes para conceituar o fenômeno. Ainda que estes se refiram a alguns estudos pioneiros no campo, conforme apresentado anteriormente, todos estes partem de uma mesma leitura da nostalgia que parece não dar conta de explicar suas dinâmicas e seus contornos contemporâneos. A visão universalista e individualista do fenômeno é incapaz de responder a perguntas como: por que estamos testemunhando um aumento do interesse de diversos grupos de consumidores pelo passado? Por que esse interesse mercadológico pela nostalgia vem ocorrendo nesse momento histórico? Como observou Brown (2018), por que essa "onda" de nostalgia que se pensava acabar após a virada do milênio não demonstra sinais de diminuição? Enfim, estudos da perspectiva cultural trazem interpretações e evidências que fragilizam o olhar sentimentalista da nostalgia.

A perspectiva cultural, por outro lado, mostra-se mais aberta a novas compreensões sobre o fenômeno ao adotar um olhar alinhado com perspectivas antropológicas, históricas e sociológicas, priorizando o contexto e as dinâmicas culturais em torno da nostalgia. No entanto, assim como a perspectiva sentimentalista, ela concebe o consumo como um processo centrado no agente humano, apesar de investigar a nostalgia realizando um esforço de integração entre estrutura e agência. Essa visão dualista, apesar de permitir a identificação de dinâmicas mais amplas e integradas do fenômeno da nostalgia, ainda assim negligencia o papel de outros elementos que atravessam o processo do consumo, como os objetos, as rotinas corporais e mentais, as competências e a linguagem, entre outros.

Ao traçar uma história do campo da Consumer Culture Theory (CCT), Askegaard e Linnet (2011) lembram que esta nasceu da visão crítica às pesquisas de comportamento do consumidor influenciadas pela Psicologia e pela Economia, argumentando que tais ciências traziam premissas que situam o consumidor em um ambiente controlado e colocam as condições estruturais do consumo em segundo plano. Contudo, os autores observam que a alternativa antropológica adotada pela CCT a partir da década de 1980 - que concebe o consumidor como um construtor de identidades reflexivo; que vive em comunidades de consumo; e que busca recursos simbólicos no mercado - apesar de enfatizar as estruturas de significados, acabou por manter o consumidor como centro do processo de consumo, desprezando outros elementos e dinâmicas. Do mesmo modo, parece que a perspectiva cultural da nostalgia, apesar de se esforçar para superar a visão universalista e cognitivista do fenômeno ao evidenciar seus contornos históricos e culturais, também caiu na mesma armadilha antropocêntrica (Reckwitz, 2002a) do início da CCT, deslocando a ênfase no indivíduo para a cultura, mas ainda concebendo o mundo social como puramente humano.

Nesse sentido, será argumentado a seguir que as Teorias da Prática podem contribuir para criar novas possibilidades de investigação do fenômeno da nostalgia ao transcenderem o dualismo estrutura-agência, olhando a integração dos diferentes elementos que participam do processo do consumo a partir das práticas como unidade de análise (Moura \& Bispo, 2019). Outra contribuição dessa abordagem está ainda em sua atenção aos objetos, não apenas com sua carga expressiva, mas como elementos que atuam materialmente na performance da vida social. $O$ tópico a seguir procura apresentar conceitos fundamentais das Teorias da Prática, bem como alguns exemplos ilustrativos dos potenciais de desenvolvimento do tema da nostalgia a partir do uso dessa abordagem.

\section{Teorias da Prática: conceitos principais}

As Teorias da Prática, apesar da alcunha "teoria", não compõem uma teoria unificada, mas um grupo de conceitos e premissas cujas principais fontes originárias são textos de diversos autores, como Pierre Bourdieu, Anthony Giddens, Jean Lave, Etienne Wenger, Theodore Schatzki, Harold Garfinkel, Michel de Certeau, Andreas Reckwitz, Davide Nicolini, Antonio Strati, Barbara Czarniawska, Silvia Gherardi e Elizabeth Shove, Mika Pantzar e Matt Watson (Gherardi, 2019; Hui et al., 2017; Nicolini, 2013). De acordo com Hui et al. (2017), ainda que estes autores possuam diferentes visões sobre a sociedade e seu funcionamento, eles são unidos pelo compartilhamento da premissa de que as práticas constituem o domínio básico do estudo das Ciências Sociais, sendo estas entendidas como conjuntos organizados de ações que se conectam e formam complexos maiores e constelações. Feldman e Worline (2016) acrescentam que as Teorias da Prática também compartilham a ideia de que as coisas (incluindo identidades, ideias, instituições, poder e bens materiais), em vez de terem o significado como características inatas de seu ser, adquirem significado à medida que são situadas em práticas sociais. 
Vale frisar que os estudos que compartilham tais premissas não formam necessariamente uma escola de pensamento. No campo dos estudos organizacionais, por exemplo, o rótulo "Estudos Baseados em Prática" agrupa uma série de abordagens com bases teóricas diversas que adotam a prática como lente para o estudo do fenômeno organizacional (Bispo, 2013). No campo dos estudos de cultura e consumo, Warde (2005) utiliza o rótulo "Teorias da Prática" para descrever os estudos que compreendem o consumo como um momento presente nas práticas sociais. Já Shove et al. (2012) e Feldman e Worline (2016), por exemplo, utilizam o rótulo "Teoria da Prática" para descrever esse grupo de premissas sobre o mundo social. Assim, considerando a diversidade de abordagens sobre a prática como unidade básica do social e o posicionamento de tal discussão no campo dos estudos do consumo, este trabalho utiliza o rótulo "Teorias da Prática", proposto por Warde (2005), para se referir ao conjunto de conceitos e premissas expostos nestes trabalhos.

De acordo com Reckwitz (2002b), uma prática pode ser uma performance individual (práxis) ou uma entidade social (praktiken). Enquanto performance individual, a prática descreve uma ação humana e se relaciona ao fazer; enquanto entidade social, uma prática significa "um tipo de comportamento rotinizado que consiste em vários elementos interligados entre si: formas de atividades corporais, mentais; coisas e seu uso; conhecimento na forma de compreensão, know-how e estados de emoção" (Reckwitz, 2002b, p. 249). Para Schatzki (1996, p. 89), as práticas são "nexos de fazeres e dizeres", dispersos no tempo e no espaço. Warde (2005) sustenta que esse nexo de fazeres e dizeres é coordenado e conectado por três elementos da prática: os entendimentos, os procedimentos e os engajamentos. Já a categorização dos elementos da prática feita por Shove et al. (2012) parece dar mais ênfase ao papel da materialidade, ao definir seus componentes nos seguintes grupos: 1) materiais: abrangendo objetos, infraestruturas, ferramentas, hardware e o próprio corpo; 2) competências: múltiplas formas de compreensão e conhecimento prático que fazem as ligações entre os elementos da prática; e 3) significados: o significado social e simbólico da participação na prática.

Tal destaque à materialidade da abordagem de Shove et al. (2012) condiz com a observação de Reckwitz (2002a) de que, enquanto as abordagens culturalistas da teoria social estudam os fenômenos sociais a partir da compreensão de que a estruturação das ações humanas é resultado de ordens simbólicas coletivas, para as Teorias da Prática, o mundo social consiste não apenas em seres humanos e suas relações intersubjetivas, mas também simultaneamente da materialidade, como o corpo, o conhecimento, a linguagem e a mente. Vale observar que a materialidade não diz respeito somente a coisas físicas, sendo ela mais uma qualidade das relações entre sujeitos e coisas do que das coisas em si (Borgerson, 2005). Essa relação entre os seres humanos e as coisas é definida por Moura e Bispo (2019) como sociomaterialidade.

Sendo a prática uma entidade social, o indivíduo atua como um "carregador" de uma prática, e não como o agente que a determina e orquestra outros elementos (Shove et al., 2012). Em outras palavras, os indivíduos são corpos/mentes que participam de práticas sociais. Assim, enquanto as abordagens culturais da teoria social baseiam-se predominantemente em uma perspectiva antropocêntrica, as Teorias da Prática tratam o indivíduo de forma descentralizada, sendo seu corpo, por exemplo, um elemento material, enquanto sua mente é tratada como um elemento de competência.

Ao integrar os elementos materiais ao mundo social, as Teorias da Prática trazem também importantes consequências para o estudo do mundo material. Enquanto as abordagens culturais compreendem o mundo material principalmente como um conjunto de coisas que portam significados culturais ou objetos de representação para os humanos, para as abordagens baseadas na Prática, as coisas devem ser compreendidas como artefatos que participam de práticas sociais e desempenham papel agêntico, o qual se expressa nos efeitos sobre outros elementos de uma prática (Borgerson, 2013). Um exemplo de estudo que investiga a agência dos objetos é o estudo de Epp e Price (2010), em que as autoras descrevem a biografia de uma mesa no contexto de uma família e demonstram que tal objeto, mesmo quando é removido das práticas familiares, continua a exercer sua agência devido à sua história no contexto familiar. Desse modo, os consumidores buscam constantemente alternativas para reincorporar esse objeto em suas práticas. Essa agência manifesta-se, por exemplo, na definição do tipo de casa a comprar ou como será a decoração de determinado cômodo para que a mesa possa fazer parte das práticas de materialidade. No momento em que ela permanece dormente, ainda assim gera mobilização dos outros elementos no sentido de modificar outras práticas familiares para seu retorno.

Reckwitz (2002b) também destaca o papel do corpo e da mente nas práticas sociais. Enquanto as abordagens culturais compreendem corpo e mente como elementos relacionados de maneira hierárquica, sendo o corpo instrumento da mente, uma abordagem baseada em prática entende que o corpo possui o papel de performar os atos comportamentais e a mente envolve rotinas de compreensão, desejo e know-how. Isso significa que uma prática envolve, além da participação do ser humano e dos elementos materiais, certas competências, as quais informam e integram os elementos da prática. 
As práticas sociais também possuem uma relação de dominância. Segundo Shove et al. (2012), uma prática dominante é aquela que sobrepõe práticas menos importantes e orientam as maneiras pelas quais as pessoas gastam seu tempo e definem as prioridades em torno das quais suas vidas são organizadas. Os autores também destacam que as práticas atravessam três fases, de modo genérico. Antes de se tornar uma prática social consolidada, isto é, com as relações entre os elementos efetivamente estabelecidas, há uma protoprática - em que os elementos demonstram potencial de ligação, mas não se encontram relacionados. De modo semelhante, quando os links entre os elementos da prática não são mais sustentados, tem-se uma ex-prática.

Vale observar que os conceitos e premissas sobre as Teorias da Prática elencados nesta seção não buscam traçar um panorama que se pretende completo e que integre as diferentes vertentes dessas teorias. Ao contrário, buscamos elencar conceitos e premissas dessas teorias que possibilitem novas leituras do fenômeno da nostalgia no campo do Marketing propondo uma abordagem sociomaterial (Moura \& Bispo, 2019) do tema. Assim, a noção compartilhada pelas Teorias da Prática de que o mundo social é composto não somente de seres humanos, tendo também as coisas - como o corpo, a mente, os objetos e as competências - papel fundamental nas dinâmicas sociais, fornece possibilidades importantes para iluminar outros aspectos da relação entre consumo e nostalgia para além dos processos cognitivos e categorias de significados culturais. Além disso, as categorias de elementos da prática, a relação de dominância entre práticas e a noção de agência compartilhada entre seres humanos e os objetos fornecem um arcabouço conceitual relevante para novas leituras sobre a nostalgia, como será exemplificado a seguir.

\section{Possibilidades de releitura da nostalgia através das teorias da prática}

Conforme argumentado anteriormente, as duas perspectivas teóricas sobre nostalgia apresentam um olhar centrado no humano, concebendo-o como centro do processo do consumo, relegando, assim, a materialidade a um segundo plano. Como, então, a nostalgia poderia ser investigada a partir da perspectiva sociomaterial das Teorias da Prática?

Primeiramente, a partir da noção de que a nostalgia é uma emoção histórica (Boym, 2001), a perspectiva da prática pode ser utilizada para estudar a nostalgia como uma prática social. Dessa maneira, focaliza não somente as representações do consumidor sobre o passado, mas também as rotinas corporais (por exemplo, expressões, comportamentos, ações) e mentais (certos modos de querer e de sentir) e as competências para se "nostalgizar", bem como o papel dos objetos nesse processo. Tal possibilidade envolveria investigar o consumo como contexto que reúne os elementos de uma prática nostálgica.

Tomemos como exemplo um consumidor que se engaja na prática de consumo de música. Enquanto corre pela manhã, esse consumidor tem o hábito de ouvir músicas pelo Spotify. Os fones conectados a seu smartphone isolam sons externos da rua movimentada, permitindo uma apreciação detalhada das músicas sugeridas pelo aplicativo na playlist "Descobertas da semana". Essa playlist é uma das preferidas desse consumidor, já que lhe oferece "novidades antigas", pois, apesar de nascido nos anos 1980, ele aprecia descobrir músicas nacionais e internacionais dos anos 1960, época que ele imagina como período de grande criatividade artística, tanto que possui em casa uma coleção de discos de vinil do período. Em casa, o consumidor possui uma vitrola que é utilizada de modo menos frequente, porém em ocasiões mais especiais, como em reuniões com amigos, comemorações familiares e quando lembra de seu avô, o qual lhe dera a maior parte de seus discos. Além disso, esse consumidor possui um filho adolescente e gosta de se reunir com ele na sala onde está sua coleção para falar das músicas e dos artistas do passado e para lhe demonstrar como manusear a vitrola e cuidar corretamente dos discos de vinil.

O exemplo anterior, apesar de abordar a nostalgia a partir da fenomenologia do sujeito, também possibilita lançar luz sobre aspectos estruturais contidos nas rotinas do consumo de música, que terminam por revelar o que seria essa prática nostálgica. Em primeiro lugar, cabe destacar a articulação não apenas da dimensão de significados em nível individual ou grupal, mas também o papel das competências e da materialidade. A respeito da materialidade, o aplicativo Spotify, que funciona mediante conexão com a internet, por meio de um sistema operacional de um smartphone, disponibiliza uma oferta imediatamente acessível de músicas do passado e também um algoritmo que seleciona e sugere músicas para o consumidor hipotético com base no que ele ouve. Isso reforça sua preferência por músicas dos anos 1960 por meio da apresentação de novas músicas do período, o que aumenta seu conhecimento e sua imaginação sobre a época. 
Os discos de vinil, por funcionarem somente em uma antiga vitrola, exigem a articulação de competências diferentes daquelas utilizadas para consumir músicas pelo Spotify. Enquanto competências relacionadas à execução de aplicativos em dispositivos móveis são articuladas para o consumo de músicas pelo Spotify, o consumo de música por meio da coleção de discos de vinil exige habilidades ligadas à identificação de lojas especializadas, ao manuseio e à operação dos equipamentos e aos cuidados de manutenção dos discos e da vitrola. A coleção de discos de vinil também envolve a articulação de comportamentos, conhecimentos e significados sociais relacionados com a prática social de colecionar. Na esfera dos significados, ainda se nota uma distinção entre ouvir música através de uma materialidade contemporânea e de uma materialidade antiga. Enquanto as novas tecnologias e competências são associadas ao lazer, ao individualismo e à eficiência, as materialidades antigas e competências a elas associadas são relacionadas pelo consumidor a ocasiões e memórias especiais e socialização.

Além disso, no exemplo anterior também podemos refletir sobre possíveis efeitos transformadores dessa prática nostálgica sobre outras práticas acopladas à prática de consumo de música. $\mathrm{O}$ encontro do consumidor hipotético com seus amigos (prática de socialização) para ouvir música (prática de lazer) tem como ponto de ligação, no exemplo, essa prática nostálgica, a qual leva o consumidor e seus amigos a compartilharem o interesse pelo passado - nesse caso, a tecnologia do vinil e as competências e os significados a ela associados. A coligação entre prática de lazer e prática de educação por meio da prática nostálgica também ocorreria quando o consumidor ensinasse seu filho sobre o uso da vitrola e sobre seus discos. No caso, o filho do consumidor hipotético participa não somente de uma prática de educação, mas também aprende a reconhecer transformações que se articulam a partir da reunião de novos objetos, significados e competências. Nesse sentido, a prática nostálgica teria o potencial de transformar determinada prática com a qual se acopla e de criar zonas de aproximação entre práticas, coligando-as temporariamente.

A lente da prática também pode contribuir para o estudo da nostalgia sob uma perspectiva histórica ao permitir o mapeamento ou a identificação de transformações de práticas sociais ao longo do tempo. As práticas articuladas junto com a prática nostálgica normalmente envolvem um esforço do consumidor para conectar elementos de ex-práticas (Shove et al., 2012), ou seja, objetos, competências e significados do passado que se encontram em estado de latência. Além disso, essa inserção de elementos abandonados de ex-práticas em práticas consolidadas poderia transformar esta última, de modo a criar uma nova prática substituta da atual ou paralela a ela. $O$ estudo de tal dinâmica, contudo, exigiria um recorte temporal relativamente amplo, pois o estabelecimento de novas relações e a quebra de links atuais costumam ser processos demorados.

No caso do consumidor de música exemplificado, podemos pensar que a própria prática de consumo de música foi transformada a partir da invenção de dispositivos que permitiam gravar as performances dos músicos. Ou seja, uma prática que era efêmera e dependia da execução da música ao vivo pelo artista, a partir da invenção do fonograma, passou a integrar elementos materiais e simbólicos de práticas familiares, por exemplo. Do mesmo modo, a criação dos videoclipes passou a possibilitar o consumo visual da música, e integrou novos elementos à prática de consumo de música, como o vídeo e a competência de apreciação cinematográfica. Assim como novas tecnologias proporcionaram transformações na prática de consumo da música, criando novas possibilidades de experiências, pode-se indagar: quais as implicações das transformações estéticas e tecnológicas desencadeadas pelo resgate de elementos de ex-práticas para a prática de consumo de música? Em síntese, como a coexistência de elementos de práticas e ex-práticas afeta o consumo?

Ainda sobre a transformação de práticas, Boym (2001) argumenta que a criação de monumentos históricos, museus e até parques temáticos são consequências da nostalgia. Podemos pensar tais espaços como repositórios que alimentam as práticas nostálgicas, mantendo vivos objetos, significados e competências, bem como suas conexões. Assim, podemos também refletir sobre como o cenário contemporâneo pode transformar a própria prática nostálgica. Uma possível questão seria: como a disponibilidade de novas tecnologias como a inteligência artificial, a realidade aumentada e a própria coletânea de dados da web pode afetar a prática nostálgica? Ou, ainda, como novas tecnologias ampliam as conexões entre a prática nostálgica e outras práticas sociais? Quais as implicações do aumento da capacidade de memória para a prática nostálgica?

Boym (2001) observa também que a nostalgia possui um caráter produtivo, pois "espacializa o tempo", produzindo categorias de passado para refletir sobre o presente e o futuro; e "temporaliza o espaço" ao combinar diferentes noções de tempo no "aqui e agora", buscando exceder o presente. Nesse sentido, a lente da prática permitiria investigar como a prática nostálgica espacializa o tempo, criando categorias temporais de passado e alocando objetos e imagens nelas. Por exemplo, quando emerge uma categoria temporal de "música dos anos 1960", cria-se um espaço de tempo que separa aquilo que é presente/moderno/contemporâneo de um passado retalhado constituído por várias categorias de passados, como "anos 1980", "anos 1950", "anos 1920", de modo que determinados objetos, imagens e competências são alocados em tais categorias, enquanto outros caem no esquecimento. 
Do mesmo modo, pode-se refletir historicamente como a prática nostálgica temporaliza o espaço no cenário contemporâneo, combinando diferentes noções de tempo em diversas práticas sociais. A prática de consumo de música ilustrada, por exemplo, combina elementos e tecnologias inovadoras (como os serviços de streaming, de armazenamento em nuvem etc.) com tecnologias que foram praticamente abandonadas (vitrola e vinil). Por que alguns elementos, mesmo com substitutos que os superam tecnologicamente, persistem nas práticas? Por que outros elementos foram totalmente abandonados? Que pistas um elemento latente deixa no interior das práticas correntes que podem indicar sua possível volta? Como a nostalgia se relaciona com elementos latentes de práticas de consumo? Enfim, as questões levantadas nesta seção ilustram apenas algumas possibilidades interpretativas a partir da concepção da nostalgia como prática social. Entretanto, as reflexões aqui realizadas pretendem indicar caminhos para pesquisas futuras sobre nostalgia no campo do Marketing que desafiem o modo atual como o campo vem estudando o fenômeno e seu lugar no consumo.

\section{CONSIDERAÇÕES FINAIS}

O presente trabalho teve como objetivo refletir e propor novas possibilidades de investigação do fenômeno da nostalgia no campo do Marketing a partir das Teorias da Prática, argumentando a favor de um alinhamento entre a investigação da nostalgia e as novas abordagens no campo do comportamento do consumidor que enfatizam aspectos sociomateriais do consumo. Ao revisitar a literatura sobre nostalgia no campo do consumidor para entender como o campo conceitua e investiga o fenômeno, foi possível identificar duas categorias de abordagens conceituais e teóricas predominantes: a sentimentalista e a cultural.

A perspectiva sentimentalista, mais antiga e predominante no campo de comportamento do consumidor, tem suas raízes em teorias sociais que focam a relação entre o consumidor e um referente (marca, produto, propaganda, outro consumidor) e os processos cognitivos de idealização, investigando principalmente os efeitos da nostalgia sobre o consumo e privilegiando a investigação no nível individual. A perspectiva cultural, relativamente mais recente e diversificada em suas investigações, compreende a nostalgia como um fenômeno cultural em movimento, que não só modifica o fenômeno do consumo como também é transformada pelo sistema de Marketing e pelas mudanças sociais e tecnológicas. Assim, a primeira contribuição do trabalho foi a organização conceitual dos estudos sobre nostalgia no campo do comportamento do consumidor, o que pode auxiliar o posicionamento conceitual de pesquisas futuras sobre o tema.

Em seguida, ao analisarmos criticamente as contribuições e limitações das duas perspectivas identificadas, argumentou-se que estas, apesar de trazerem olhares distintos, compartilham um viés agêntico, concebendo o consumidor como centro do processo de consumo, e antropocêntrico, no sentido de compreenderem o mundo social como exclusivamente humano, excluindo o mundo material ou tratando-o como um mundo à parte de objetos a serviço de representações e usos dos consumidores. Com base na apreciação crítica das duas perspectivas e discussão conceitual das Teorias da Prática, foi possível contribuir também através da criação de propostas de releitura da nostalgia por meio de exemplos de investigações possíveis sobre a relação entre nostalgia e consumo utilizando as Teorias da Prática como lente.

Cabe ressaltar, ainda, as limitações do presente trabalho. Em primeiro lugar, a organização conceitual da literatura não esgota as temáticas e abordagens da nostalgia presentes no campo, pois não quantifica ou qualifica a literatura com base em critérios bibliométricos ou sistemáticos. Por outro lado, acredita-se que a literatura selecionada e revisada, por ser predominantemente originária de periódicos internacionais de alto impacto na área de Marketing e de grande relevância para as pesquisas de comportamento do consumidor, permite estabelecer um panorama das principais abordagens conceituais do tema, uma vez que os trabalhos publicados em periódicos dessa natureza abrem ou influenciam novos caminhos de pesquisa.

Cabe também ressaltar que os exemplos utilizados para ilustrar possíveis aplicações das Teorias da Prática para investigar a nostalgia não esgotam as interpretações e associações possíveis ao se adotar tal perspectiva. Os exemplos foram utilizados, principalmente, para ilustrar alguns conceitos das Teorias da Prática que consideramos aplicáveis à compreensão do fenômeno da nostalgia no contexto do consumo, desafiando as perspectivas atuais sobre o tema. Como argumentado no texto, os próprios conceitos das Teorias da Prática apresentados e discutidos não são capazes de captar toda a complexidade dessa lente, a qual se encontra em construção.

Espera-se, dessa forma, que este trabalho contribua para renovar as pesquisas sobre a nostalgia em Marketing, indicando percursos investigativos viáveis e relevantes para a comunidade de pesquisadores de comportamento do consumidor ao propor novas leituras sobre um fenômeno que, mesmo se referindo ao passado, parece nunca ter sido tão contemporâneo. 


\section{REFERÊNCIAS}

Angé, O., \& Berliner, D. (2015). Anthropology and nostalgia. New York, NY: Berghahn Books.

Anspach, C. K. (1934). Medical Dissertation on Nostalgia by Johannes Hofer, 1688. Bulletin of the Institute of the History of Medicine, 2(6), 376-391.

Areni, C. (2019). Ontological security as an unconscious motive of social media users. Journal of Marketing Management, 35(1-2), 75-96.

Armstead, C. S., \& McKinney, E. (2019, dezembro). The role of nostalgia in retro sewing. Journal of Consumer Culture. Recuperado de https://doi.org/10.1177/1469540519893102

Arnould, E., \& Thompson, C. (2005, março). Consumer Culture Theory (CCT): twenty years of research. Journal of Consumer Research, 31, 868-882.

Askegaard, S., \& Linnet, J. T. (2011, dezembro). Towards an epistemology of consumer culture theory: Phenomenology and the context of context. Marketing Theory, 11(4), 381-404.

Belk, R. W. (1990). The role of possessions in constructing and maintaining a sense of past. Advances in Consumer Research, 17(1), 669-676.

Belk, R. W. (1991). Possessions and the sense of past. Highways and buyways: naturalistic research from the consumer behavior odyssey. Provo, UT: Association for Consumer Research.

Billboard. (2020, agosto). Future Nostalgia - Dua Lipa. Recuperado de https://www.billboard.com/charts/billboard-200

Bispo, M. (2013). Estudos baseados em prática: conceitos, história e perspectivas. Revista Interdisciplinar de Gestão Social, 2(1), 13-33.

Borgerson, J. L. (2005). Materiality, agency, and the constitution of consuming subjects: insights for consumer research. Advances in Consumer Research, 32, 439-443.

Borgerson, J. L. (2013). The flickering consumer: new materialities and consumer research. Research in Consumer Behavior, 15, 125-144.

Boym, S. (2001). The future of nostalgia. New York, NY: Basic Books, 2001.

Braga, C., \& Suarez, M. (2018). Teoria Ator-Rede: novas perspectivas e contribuições para os estudos de consumo. Cadernos EBAPE.BR, 16(2), 218-231.

Brown, S. (2018). Retro Galore! Is there no end to nostalgia? Journal of Customer Behaviour, 17(1), 9-29.

Brown, S., Hirschman, E. C., \& Maclaran, P. (2001). Always Historicize! Researching marketing history in a post-historical epoch. Marketing Theory, 1(1), 49-89.

Brown, S., Kozinets, R. V., \& Sherry, J. F. (2003). Teaching old brands new tricks: retro branding and the revival of brand meaning. Journal of Marketing, 67, 19-33.

Brunk, K. H., Giesler, M., \& Hartmann, B. J. (2018). Creating a consumable past: how memory making shapes marketization. Journal of Consumer Research, 44(6), 1325-1342.

Campbell, C. (1987). The romantic ethic and the spirit of modern consumerism. Oxford, UK: Basil Blackwell.
Cervellon, M. C., \& Brown, S. (2018). Reconsumption reconsidered: redressing nostalgia with neo-burlesque. Marketing Theory, 2012, 1-20.

Cross, G. (2017, March). Nostalgic collections. Consumption Markets \& Culture, 20(2), 101-106.

Davari, A., lyer, P., \& Guzmán, F. (2017, novembro). Determinants of brand resurrection movements: why consumers want dead brands back? European Journal of Marketing, 51(11/12), 1896-1917. Recuperado de https://doi.org/10.1108/EJM-02-2016-0096

Davis, F. (1979). Yearning for yesterday: a sociology of nostalgia. New York, NY: Free Press.

Delanda, M. (2006). A new philosophy of society: assemblage theory and social complexity. Nova York, NY: Continuum.

Enano, V. L. (2019, março 10). O negócio da nostalgia. El País. Recuperado de https://brasil.elpais.com/brasil/2019/03/05/ eps/1551786074_152123.html

Epp, A. M., \& Price, L. L. (2010). The storied life of singularized objects: forces of agency and network transformation. Journal of Consumer Research, 36(5), 820-837.

Feldman, M., \& Worline, M. (2016). The practicality of practice theory. Academy of Management Learning \& Education, 15(2), 304-324.

Ford, J. B. Merchant, A., Bartier, A. L., \& Friedman, M. (2018, fevereiro). The cross-cultural scale development process: the case of brand-evoked nostalgia in Belgium and the United States. Journal of Business Research, 83, 19-29.

Garrido, S., \& Davidson, J. W. (2019). Music, nostalgia and memory. London, UK: Palgrave Macmillan, 2019.

Gehlen, A. (2017). A felicidade evadida. Uma interpretação da nostalgia. História da Historiografia, 10(23), 142-152.

Gherardi, S. (2019). How to conduct a practice-based study: problems and methods ( $2 \mathrm{a}$ ed.). Cheltenham, UK: Elgar.

Goulding, C. (2001). Romancing the past: heritage visiting and the nostalgic consumer. Psychology and Marketing, 18(6), 565-592.

Goulding, C., \& Derbaix, M. (2019). Consuming material authenticity in the age of digital reproduction. European Journal of Marketing, 53(3), 545-564.

Hamilton, K., Edwards, S., Hammil, F., Wagner, B., \& Wilson, J. (2014, março). Nostalgia in the twenty-first century. Consumption Markets \& Culture, 17(2), 101-104.

Higson, A. (2014, março). Nostalgia is not what it used to be: heritage films, nostalgia websites and contemporary consumers. Consumption Markets \& Culture, 17(2), 120-142.

Holak, S. L., \& Havlena, W. J. (1998). Feelings, fantasies, and memories: an examination of the emotional components of nostalgia. Journal of Business Research, 42(97), 217-226.

Holbrook, M. B. (1993). Nostalgia and consumption preferences: some emerging patterns of consumer tastes. Journal of Consumer Research, 20(2), 245-256.

Holbrook, M. B., \& Schindler, R. M. (1991). Echoes of the dear departed past: some work in progress on nostalgia advances in consumer 
research. Recuperado de http://www.acrwebsite.org/search/viewconference-proceedings.aspx?ld=7181

Holbrook, M. B., \& Schindler, R. M. (1996). Market segmentation based on age and attitude toward the past concepts, methods, and findings concerning nostalgic influences on customer tastes. Journal of Business Research, 37, 27-39.

Huang, X., Huang, Z., \& Wyer, R. S. (2016, outubro). Slowing down in the good old days: the effect of nostalgia on consumer patience. Journal of Consumer Research, 43(3), ucw033.

Hui, A., Schatzki, T., \& Shove, E. (2017). The nexus of practices: connections, constellations, practitioners. London, UK: Routledge.

Jameson, F. (1991). Postmodernism, or, the cultural logic of late capitalism. London, UK: Verso.

Kessous, A., Roux, E., \& Chandon, J. L. (2015). Consumer-brand relationships: a contrast of nostalgic and non-nostalgic brands. Psychology \& Marketing, 32(2), 187-202.

Khoshghadam, L., Kordrostami, E., \& Liu-Thompkins, Y. Experiencing nostalgia through the lens of life satisfaction. European Journal of Marketing, 53(3), 524-544, 2019.

Lasaleta, J. D., Sedikides, C., \& Vohs, K. D. (2014). Nostalgia weakens the desire for money. Journal of Consumer Research, 41(3), 713-729.

Latour, B. (1988). Mixing humans and nonhumans together: the sociology of a door-closer. Social Problems, 35(3), 298-310.

Loveland, K. E., Smeesters, D., \& Mandel, N. (2010). Still preoccupied with 1995: the need to belong and preference for nostalgic products. Journal of Consumer Research, 37(3), 393-408.

Maclaran, P., \& Brown, S. (2005). The center cannot hold: consuming the utopian marketplace. Journal of Consumer Research, 32(2), 311-323.

McCracken, G. (1988). Culture and consumption: new approaches to the symbolic character of consumer goods and activities. Bloomington, Indiana: Indiana University Press.

Moura, E. O., \& Bispo, M. S. (2019). Sociomateriality: theory, methodology, and practice. Canadian Journal of Administrative Science. Recuperado de https://doi.org/10.1002/cjas.1548
Muehling, D. D., Sprott, D. E., \& Sultan, A. J. (2014). Exploring the boundaries of nostalgic advertising effects: a consideration of childhood brand exposure and attachment on consumers' responses to nostalgia-themed advertisements. Journal of Advertising, 43(1), 73-84

Nicolini, D. (2013). Practice theory, work, \& organization: an introduction. Oxford, UK: Oxford University Press.

Pickering, M., \& Keightley, E. (2006). The modalities of nostalgia. Current Sociology, 54(6), 919-941.

Reckwitz, A. (2002a). The status of the "material" in theories of culture: from "social structure" to "artefacts". Journal for the Theory of Social Behaviour, 32(2), 195-217.

Reckwitz, A. (2002b). Toward a theory of social practices: a development in culturalist theorizing. European Journal of Social Theory, 5(2), 243-263.

Routledge, C. (2016). Nostalgia: a psychological resource. London, UK: Routledge.

Schatzki, T. (1996). Social practices: a wittgensteinian approach to human activity and the social. Cambridge, UK: Cambridge University Press.

Shove, E., Pantzar, M., \& Watson, M. (2012). The dynamics of Social Practice. Los Angeles, CA: Sage.

Stern, B. B. (1992). Historical and personal nostalgia in advertising text: The fin de siecle effect. Journal of Advertising, 21(4), 11-22.

Suvinil. (2020). Verde nostalgia. Recuperado de https://www.suvinil. com.br/cor/verde-nostalgia-d063

Warde, A. (2005). Consumption and theories of practice. Journal of Consumer Culture, 5(2), 131-153.

Zhou, L., Wang, T., Zhang, Q. \& Mou, Y. (2013). Consumer insecurity and preference for nostalgic products: evidence from China. Journal of Business Research, 66(12), 2406-2411.

Zhou, X., Wildschut, T., Sedikides, C., Shi, K., \& Feng, C. (2011). Nostalgia: the gift that keeps on giving. Journal of Consumer Research, 39(1), 39-50.

Flávio Medeiros Henriques

ORCID: https://orcid.org/0000-0002-7213-509X

Doutorando em Administração pelo Instituto COPPEAD de Administração da Universidade Federal do Rio de Janeiro (UFRJ); Professor de Marketing no Instituto Federal de Educação, Ciência e Tecnologia do Rio de Janeiro (IFRJ).E-mail: flavio.medeiros@coppead.ufrj.br

Maribel Carvalho Suarez

ORCID: https://orcid.org/0000-0001-9736-5273

Doutora em Administração pela Escola de Negócios (IAG) da Pontifícia Universidade Católica do Rio de Janeiro (PUC-Rio), com estágio pós-doutoral pela Schulich School of Business - Canadá; Professora Adjunta do Instituto COPPEAD de Administração da Universidade Federal do Rio de Janeiro (UFRJ). E-mail: maribels@coppead.ufrj.br 